\title{
COVID-19-Vaccination Prioritization in Patients with Cancer
}

Attapon Cheepsattayakorn ${ }^{1,2^{*}}$, Ruangrong Cheepsattayakorn ${ }^{3}$ and Thanom Jewsuebpong ${ }^{1}$

${ }^{1}$ Faculty of Medicine, Western University, Pathumtani Province, Thailand

${ }^{2} 10^{\text {th }}$ Zonal Tuberculosis and Chest Disease Center, Chiang Mai, Thailand

${ }^{3}$ Department of Pathology, Faculty of Medicine, Chiang Mai University, Chiang Mai, Thailand

\section{*Correspondence authors}

Attapon Cheepsattayakorn, $10^{\text {th }}$ Zonal Tuberculosis and Chest Disease Center 143 Sridornchai Road Changklan Muang Chiang Mai Thailand

Submitted : 23 Sept 2021 ; Published : 4 Nov 2021

In patients with cancer, SARS-CoV-2 (COVID-19) can contribute to increasing morbidity and mortality and decreased survival was found in patients with hematological and intrathoracic malignancies, poor performance status, comorbidities, and increased age. Patients with hematological malignancies who were treated with stem cell transplantation and anti-CD-20 antibody demonstrated lower rates of seroconversion, compared to COVID-19-infected-cancer patients. Patients with hematological malignancies might have substantially compromised B-cell and T-cell responses. These study results indicated that following COVID-19 vaccination, overall high seroconversion rates could be anticipated in cancer patients due to different mechanisms and degrees of immune suppression, such as cell therapies (particularly chimeric antigen receptor (CAR)-T cell), anti-CD-20 antibody (B-cell depleting) therapies, stem cell transplantation, immunosuppressive effects of corticosteroid treatment, and cytotoxic-chemotherapy-bone-marrow-suppressive effects in certain subgroups of cancer patients. Currently, there are lacking data in cancer patients in protection following SARS-CoV-2 (COVID-19) infection, reinfection by various SARS-CoV-2 (COVID-19) variants, or COVID-19 vaccination although mucosal surface antigens (e.g. IgA and protective T-cell responses) might be similarly important in protection from natural SARS-CoV-2 (COVID-19) infection. The association of carcinogenesis with genomic information encoding vaccines, particularly with very-transient-intracellular-presence-COVID-19-mRNA vaccines is likely very low.

In conclusion, for continuation of the quality of oncological care, cancer patients on clinical trials should be prioritized for COVID-19 vaccination that do not affect the eligibility of the clinical trials.

Copyright: (C2021 Attapon Cheepsattayakorn. This is an open-access article distributed under the terms of the Creative Commons Attribution License, which permits unrestricted use, distribution, and reproduction in anymedium, provided the original author and source are credited. 